\title{
Editorial
}

\section{Failed intubation in the parturient}

The article by Davies et al. ${ }^{1}$ attempts to outline a management plan for one of the most critical events in obstetrics, that of a failed intubation during general anaesthesia for delivery. In the authors' historical review, which was based on data from England, Scotland and Wales, the incidence of failed intubation in this circumstance is extremely low. It is unfortunate that the North American experience is not readily available. This limitation may reflect the difficulty in obtaining accurate morbidity and mortality data at the present time.

Previous studies from other countries support a low frequency of difficulty. Tiret et al. ${ }^{2}$ carried out a prospective review of complications associated with anaesthesia in France from 1978-1982. They examined a sample from 198,103 anaesthetics performed in 460 public hospitals. Unfortunately, complications related to an occurrence during obstetrics were not specified, but they indicated that a complication during intubation was a factor in $\mathbf{1 4}$ cases resulting in one comatose patient but no deaths. From Australia, the 1986 report of the Victorian Consultative Council on Anaesthetic Mortality and Morbidity $^{3}$ contains a review of 69 cases. There were four cases of failed intubation, two of which occurred during caesarean section for patients with pre-eclampsia. These patients were subsequently intubated following a plan of action, thus averting morbidity. In Canada, the CMPA ${ }^{4}$ has reviewed all closed claims since 1980 which deal with failed intubation in obstetrics. Of seven cases, three resulted in death, one had severe brain damage after blind nasal intubation and one recovered after aspiration. Of the two remaining patients, one was successfully intubated by a second anaesthetist and the other survived following an emergency tracheotomy. Only three cases involved legal action. When one considers the experience of the anaesthetist and the management of failed intubation it is important to examine Lyons' paper. ${ }^{5}$ Of the eight cases of difficult intubation in 2,331 general anaesthetics, seven were converted to epidural anaesthesia and one delivered vaginally after general anaesthesia was abandoned. Two

Department of Anaesthesia, The Hospital for Sick Children, Toronto. of the eight cases involved a consultant and six involved either a registrar or senior house officer. This experience would support the need for failed intubation drills early in postgraduate training programs. Nevertheless, despite the low incidence, anaesthetists must not be complacent. A failure to intubate under any circumstances can have disastrous consequences.

Davies and her colleagues review the essential examination necessary to evaluate the airway before anaesthesia and make recommendations reganding specific equipment which should be available in the operating room. They also, in considerable detail, outline the necessary steps to secure the airway and to confirm accurate placement of the tracheal tube. Finally, they offer an algorithm in the event of failure. While their advice is reasonable and fundamental in its approach, the final remarks regarding surgical intervention including needle and catheter cricothyrotomy and high pressure ventilation techniques demand a note of caution. Without proper placement of the catheter, the application of high pressure to the airway by inexperienced hands can lead to considerable complications. ${ }^{6,7}$ While the extreme urgency of the situation is recognized together with the necessity to salvage the airway, it is important to emphasize that the operator must be familiar with the limitations of these techniques which may be necessary only on very rare occasions. It is important to remember that in most cases the anaesthetic can be terminated or other intubation methods may be successful. Perhaps the addition of an oesophageal obturator airway to the protocol may provide a less complicated and safer alternative ${ }^{8}$ In the final analysis it is important to stress the need for careful preoperative evaluation of the airway as a form of preventative medicine. ${ }^{9}$

From a medicolegal point of view, publication of practice management articles raises a number of questions and concerns. The recent anaesthetic literature has been replete with articles on risk management which purport to show the way in a very authorative fashion. Davies' effort is another contribution. While it appears in the scientific journal of the Canadian Anaesthetists' Society, it does not represent an official CAS position. It represents one group's viewpoint. While their advice may be reasonable, 
its validity and application remain open to competing expert opinion. ${ }^{10}$ This conflict could require judicial determination in the context of a law suit following expert testimony and cross-examination. During the past few years, the CAS along with other official organizations, has published guidelines to practice and in some cases, standards of practice. These officially sanctioned documents, proposed by persons with recognized authority and expertise, are well known to the legal profession and as a result, carry significant weight in the jucicial process as the court attempts to evaluate a standard of care by a defendant in a medico-legal action. However, practical management articles play an important role in continuing medical education by reviewing certain difficult clinical situations and informing and stimulating the practitioner to enable him to expand his knowledge and develop contingency plans.

\section{Acknowledgement}

The author is grateful to Mr. Colin Campbell, McCarthy \& McCarthy for his review and assistance with the manuscript.

\section{Femme enceinte et intubation difficile}

L'article de Davies et al. ${ }^{1}$ tente de décrire un plan d'action pour l'un des événements le plus critique en obstétrique, celui d'une intubation manquée durant l'anesthésie générale pour accouchement. Dans la revue historique de l'auteur bašée sur des données d'Angleterre, d'Ecosse et de Wales, l'incidence d'intubation manquée dans ces circonstances est extrêmement basse. Il est malheureux que l'experience nord-américaine ne soit pas facilement disponible. Cette limitation peut être due présentement à la difficulté d'obtenir des données précises sur la morbidité et la mortalité.

D'autres études publiées dans d'autres pays peuvent confirmer la fréquence basse de cet incident. Tiret $e t \mathrm{al}^{2}$ dans une étude de prospective sur les complications associées à l'anesthésie en France de 1978-1982, ont examiné 198103 actes anesthésiques accomplis dans 460 hôpitaux publics. Malheureusement les complications obstétricales n'ont pas été spécifiées, mais Tiret indique qu'une complication lors de l'intubation était un facteur dans 14 cas incluant un coma et aucun décès.

D'Australie en 1986, le rapport de la Victorian Consultative Concil on Anaesthetic Mortality and Morbidity ${ }^{3}$ contient une revue de 69 cas. Il y a eu quatre cas d'intubation manquée deux desquels sont survenus lors d'une césarienne pour des patientes se présentant pour pré-éclampsie. Heureusement ces patientes furent intubées selon un protocole déterminé évitant ainsi une morbidité significative.

Au Canada la $\mathrm{CMPA}^{4}$ a revu tous les procès depuis 1980 en rapport avec une intubation manquée en obstétrique. Des sept cas étudiés trois ont abouti à un décès, un à un dommage cérébral sévère après une intubation nasale aveugle et un a recouvert après aspiration. Des deux autres patientes l'une a été intubée avec succès par un autre anesthésiste et l'autre a survécu après une trachéotomic d'urgence. Seulement trois cas furent amenés en cours.

Quand on consière l'expérience de l'anesthésiste et la conduite lors d'une intubation difficile, il est important d'examiner l'étude de Lyons. ${ }^{5}$ Dans les cas d'une intubation manquée lors de 2331 anesthésies générales, sept ont été converties en anesthésie épidurale et une accoucha par voie vaginale lorsque l'anesthésie générale fut abandonnée. Deux des huit cas ont impliqué un consultant et six ont impliqué un interne ou un résidant. Cette expérience suggère la nécessité d'inclure des exercices d'intubation manquée tôt dans les programmes d'enseignement post-gradué.

Néanmoins, malgré l'incidence basse un anesthésiste ne doit pas assumer une attitude indifférente. Une intubation difficile peut en toutes circonstances avoir des conséquences désastreuses.

Davies et ses collègues ont revu les examens essentiels nécessaires pour évaluer les voies aériennes en période pré-opératoire afin de faire des recommandations concernant certains equipements spécifiques qui devraient ére disponibles en salle d'opération. Elle a aussi défini en détail les étapes nécessaires afin d'assurer les voies aériennes libres et confirmer l'emplacement exact du tube endotrachéal. Finalement elle offre un algorithme à suivre au cas où l'intubation est impossible. Alors que son conseil est raisonnable et fondamental dans son approche, les remarques finales concernant l'intervention chirurgicale avec l'aiguille et le cathéter de cricothyrotomie ainsi que les techniques de ventilation à haute pression demandent une note de precaution. Sans l'emplacement précis du cathéter l'application d'une haute pression aux voies aériennes par des mains inexpérimentées peut amener des complications importantes. ${ }^{6,7}$ Alors que l'extrême urgence de la situation est reconnue, il est important de mettre l'emphase que l'opérateur doit être familier avec les limitations de ces techniques qui sont nécessaires uniquement dans des occasions très rares.

Il est important de se rappeler que dans la majorité des cas, l'anesthésie peut être cessée ou une autre méthode 
d'intubation peut être accomplie avec succès. Peut-être l'addition d'un obturateur oesophagien au protocole pourrait fournir une alternative moins compliquée et sécuritaire. $^{8}$

Dans l'analyse finale, il est important de mettre l'emphase sur la necessité d'une évaluation pré-opératoire des voies aériennes comme une forme de médecine préventive. ${ }^{9}$

Au point de vue médico-légal la publication d'articles de "conduite pratique " soulèvent un nombre de questions. La littérature anesthésique récente est pleine d'articles où les auteurs décrivent d'une façon autoritaire la conduite à tenir dans tel ou tel cas. L'effort de Davies en est un exemple. Alors qu'il apparaît dans le journal scientifique officiel de la Société Canadienne des Anesthésistes, il ne représente pas la position officielle de cette Société. Il représente le point de veu d'un groupe. Alors que leurs conseils peuvent être raisonnables, sa validité et son application restent à prouver par l'opinion des experts. ${ }^{10} \mathrm{Ce}$ conflit requiert un avis juridique dans le contexte d'une poursuite après témoignages d'experts et contre-examens. Durant les dernières années la S.C.A. ainsi que d'autres organisations officielles ont publié des directives et dans certains cas des normes de pratique. Ces documents officiels proposés par des personnes dont l'autorité et l'expertise sont reconnues, sont admis par la profession légale et ainsi amènent un poids significatif au processus judiciaire quand la cour veut évaluer les normes de soins lors d'une action médico-légale. Cependant les articles de "conduite pratique » jouent un rôle important dans l'éducation médicale continue en revisant certaines situations cliniques difficiles informant et stimulant le praticien afin d'étendre ses connaissances de base et de développer des plans d'urgence. On doit continuer à supporter de telles activités par nos collègues. Ces efforts sont substantiels pour nous insister à atteindre des normes élevées de soins pour nos patients.

\section{References}

1 Davies $J M$, Weeks MB, Crone LA, Pavlin E. Failed intubation in the parturient. Can J Anaesth 1989 (in this issue).

2 Tiret $I$, Hatton $F$. Complications associated with anaesthesia - a prospective survey in France. Can Anaesth Soc J 1986; 33: 336-44.

3 Paul JD, Robinson GD. Fourth report of the Victorian Council on Anaesthetic Mortality and Morbidity. 1986.

4 Lee S. Canadian Medical Protective Association. Personal communication.

5 Lyons G. Failed intubation. Anaesthesia 1985; 40: 159-62.

6 Mette PJ. Avoiding complications during jet ventilation. Anesthesiology 1980; 52: 451-2.

7 Weymuller EA, Paugh D, Pavlin EG, Cummings $C W$. Management of difficult airway problems with percutaneous transtracheal ventilation. Ann Otol Rhinol Laryngol 1987; 96: 34-7.

8 Donan N, Tweed A, Dushfsky S, Guttormson B. The esophageal obturator airway: an appraisal. Can Anaesth Soc J 1983; 30: 194-200.

9 Samsoon GLT, Young JRB. Difficult tracheal intubation: a retrospective study. Anaesthesia 1987; 42: 487-90.

10 Sharpe $G$. The Law and Medicine in Canada. 1987. 2nd Edition. Butterworths. 